\title{
Corps laïques ou corps religieux ? Une analyse des manuels scolaires de morale au XXe siècle
}

\section{Jacques Gleyse}

\section{(2) OpenEdition \\ 1 Journals}

\section{Édition électronique}

URL : http://journals.openedition.org/trema/2735

DOI : 10.4000/trema.2735

ISSN : 2107-0997

\section{Éditeur}

Faculté d'Éducation de l'université de Montpellier

\section{Édition imprimée}

Date de publication : 1 avril 2012

Pagination : $42-71$

ISSN : 1167-315X

\section{Référence électronique}

Jacques Gleyse, "Corps laïques ou corps religieux? Une analyse des manuels scolaires de morale au XXe siècle », Tréma [En ligne], 37 | 2012, mis en ligne le 01 avril 2014, consulté le 21 avril 2019. URL http://journals.openedition.org/trema/2735; DOI : 10.4000/trema.2735

Ce document a été généré automatiquement le 21 avril 2019

Trema 


\title{
Corps laïques ou corps religieux? Une analyse des manuels scolaires de morale au XXe siècle
}

\author{
Jacques Gleyse
}

1 La question de la laïcité a récemment agité le microcosme politique français tout autant que le monde scolaire. De manière récurrente ce sujet a été mis en avant par ceux qui parfois respectent le moins les principes de séparation des églises de l'État instauré par la loi du 9 décembre 1905 en France.

Le personnel politique notamment, au plus haut sommet de l'État, se rendant devant les médias, dans des lieux de culte es qualité ou se «signant» publiquement

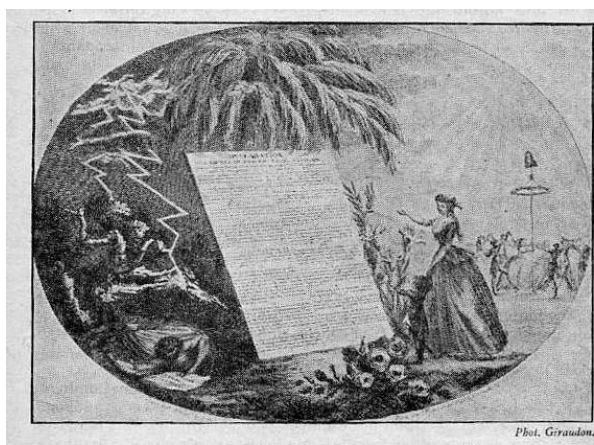

La Déclatation des Droits de l'Homag ex du Citoven.

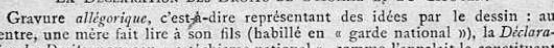
centre, une mère fait lire a son fils (habilic en " garde national m), la Diclara-
tion des Droits, nouveau a catéchisme national ", comme lappelait le constituant

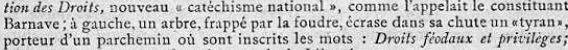
porteur dun parchemin ou sont inscrits les mots : Droits fídaux et privileges,
a droite, ronde autour du \& bonnet de la Liberté $n$. transgressent de facto la dite loi. La création récente (2010) d'une Maison universelle de la laïcité, à Paris par une association de Défense de la Laïcité, s'est posée comme un contre-feu à ces événements.

D'autres incidents ont focalisé l'attention des politiques dans les années précédentes : le voile porté, dans l'enceinte de leur établissement, par quelques rares collégiennes ou lycéennes. La burqa revêtue dans l'espace public et au volant. Les prières dans les rues, faute de place dans les mosquées. Tout cela posait pour certains, au-delà des coutumes culturelles, traditionalistes ou tribales, la question de la séparation de l'espace public et de l'espace privé, celle également du laïcus et du clericus. Le clerc religieux, qu'il soit musulman, catholique, juif, protestant ou bouddhiste prenait trop de place face au laïque séculier dans une République qui s'affirme « indivisible, démocratique, laïque et sociale ». 
3 Très récemment, un autre débat vient toucher l'espace scolaire, via son ministre de tutelle, Luc CHÂTEL : faut-il réintroduire les leçons de morale à l'école?

Dans cet article, ces deux thèmes vont être rapprochés au travers d'une histoire scolaire des rapports au corps et des représentations des comportements corporels dans les manuels scolaires de morale.

4 Si l'on observe l'histoire de l'école primaire, celle-ci est laïque, gratuite et obligatoire depuis les lois FERRY de 1881 et 1882. Dans cette optique interroger cette institution au regard du principe de la laïcité, c'est-à-dire du principe de séparation des églises et de l'État semble sans objet. Et pourtant, nous verrons qu'aujourd'hui comme hier, cette affirmation ne va pas de soi.

De même, la morale a fait partie pendant très longtemps des programmes scolaires et elle peut être encore enseignée de nos jours sous la forme de l'éducation civique. Des textes récents le rappellent du moins pour ce qui concerne l'école primaire.

\section{Programmes officiels et morale laïque?}

Les programmes du BO hors série $\mathrm{n}^{\mathrm{o}} 3$ du 19 juin 2008 proposent une :

« Reprise des grandes lignes des institutions et de la vie démocratique, des grandes lignes des programmes d'éducation civique (liens avec l'histoire et la géographie) :

- Étude des institutions et de la vie démocratique

- Valeurs et symboles de la République

- L’Union européenne et la francophonie

- Nouvel accent mis sur un enseignement de la morale. Des pratiques. Des références et des valeurs.

Plus précisément encore, les programmes affirment pour ce qui est de l'instruction civique et morale, que :

«Les élèves apprennent les règles de politesse et du comportement en société. Ils acquièrent progressivement un comportement responsable et deviennent plus autonomes.

1/ Ils découvrent les principes de la morale, qui peuvent être présentés sous forme de maximes illustrées et expliquées par le maître au cours de la journée : telles que "La liberté de l'un s'arrête où commence celle d'autrui", "Ne pas faire à autrui ce que je ne voudrais pas qu'il me fasse", etc. Ils prennent conscience des notions de droits et de devoirs.

2/ Ils approfondissent l'usage des règles de vie collective découvertes à l'école maternelle: telles l'emploi des formules de politesse ou du vouvoiement. Ils appliquent les usages sociaux de la politesse (ex. : se taire quand les autres parlent, se lever quand un adulte rentre dans la classe) et coopèrent à la vie de la classe (distribution et rangement du matériel).

3/ Ils reçoivent une éducation à la santé et à la sécurité. Ils sont sensibilisés aux risques liés à l'usage de l'internet. Ils bénéficient d'une information adaptée sur les différentes formes de maltraitance.

4/ Ils apprennent à reconnaître et à respecter les emblèmes et les symboles de la République (la Marseillaise, le drapeau tricolore, le buste de Marianne, la devise "Liberté, Égalité, Fraternité"). "

7 Ces textes se distinguent assez nettement de ceux qui les précédent, spécifiquement au point 1 , en proposant d'enseigner à nouveau la morale sous forme de maximes, autrement dit en revenant à une école de Jules FERRY fantasmée ou fantasmatique (puisque l'Instruction publique propose d'utiliser des méthodes actives dès le 20 juin 1923 et, par 
exemple, proscrit les châtiments corporels dès, a minima, 1887). Pour le reste, les contenus sont à peu près identiques à ceux des textes précédents.

Toutefois, les Instructions de 1995 ne proposaient pas du tout la même manière d'enseigner «la morale». D'une part, il n'y était pas question de morale mais «d'Éducation civique » et, d'autre part, elles expliquaient que : «C'est à partir de la vie de la classe que l'enfant découvre les règles de la vie en société, les valeurs qui la fondent et fait l'apprentissage de sa propre responsabilité ». Autrement dit il n'y était pas question de pratiques « artificielles » (telles les maximes) déconnectées de la vie réelle de l'enfant ou de connaissances difficiles à transformer en savoirs. Plus précisément encore, on y affirmait au sujet de "La vie en commun », qu'elle devait être " une pratique réfléchie au cours des diverses activités de la classe et de l'école», donc un vécu quotidien, non déconnecté des autres activités scolaires et de la vie de l'enfant. Elles affirmaient poursuivre trois objectifs :

« Respect de la personne, de soi et des autres

Mise en œuvre des règles d'hygiène, de sécurité, de tenue et justification de cellesci ; éducation à la santé

Prise de conscience du devoir de respecter les autres et du droit au respect pour soi-même, dans l'identité, la personnalité, l'intégrité physique, les biens et l'expression de la pensée de chacun. Respect du bien commun et du cadre de vie.

Éducation à la consommation.

Initiation à la gestion de l'environnement, cadre de vie des hommes et bien commun de tous.

Prise de conscience des règles de la vie en commun.

Prise de conscience des règles de la vie commune dans la classe et dans l'école (prise de parole, camaraderie, entraide, coopération)».

Il n'y était pas question d'apprendre des maximes hors du contexte du vécu de l'enfant et du vécu scolaire. Autrement dit les méthodes actives étaient privilégiées par rapport aux méthodes uniquement transmissives et passives.

Cependant, dans les deux cas, on constate que si les méthodes divergent, le fond lui reste relativement stable. On note particulièrement que la question de la laïcité n'est qu'à peine évoquée et que ce qui semble fonder la morale c'est un certain nombre de comportements, plus ou moins inculqués, sociaux et culturels. Ce sont aussi des micropouvoirs (FOUCAULT, 2002) ou des biopouvoirs, des systèmes de contrôles exercés sur les corps où le «silence et l'immobilité ", plus que jamais après 2008, semblent la règle. Il reste alors à savoir si hors de la question des méthodes pédagogiques le modèle corporel qui est enseigné au travers de la morale est un modèle laïc ou religieux et dans la deuxième optique à quelle religion appartient-il ?

\section{Les manuels de morale étaient-ils laïcs ?}

11 Lorsque l'on fait l'archéologie du système scolaire de Jules FERRY, comme le fait Michel FOUCAULT dans Surveiller et punir, on ne peut que constater que celui-ci a été fondé sur le prêche ecclésiastique puisqu'il est issu des écoles des Frères de Jean-Baptiste DE LA SALLE et sur le fait des fabriquer des " ouailles pour l'église ». On comprend dès lors pourquoi, au moins entre 1887 et 1923 quand l'enseignement par « l'aspect » est privilégié, le silence et l'immobilité des corps sont probablement de mise.

Michel FOUCAULT en 1975 (p. 141 à 171) citait Jean-Baptiste DE LA SALLE afin d'expliquer le processus disciplinaire mis en œuvre dans l'école républicaine. Il décrivait dans le 
chapitre consacré aux "alignements » et aux «corps dociles » comment, en 1720, on décrivait le corps comme devant être « docile dans ses moindres opérations » (FOUCAULT, 1975, p. 145) et comment on devait appliquer :

« Des principes utilisés naguère pour façonner des ouailles pour l'église et des sujets pour le Roi. » public, en France, connaissent les quelques expressions illustratives qui suivent ou, du moins, des injonctions morales similaires.

"On risque de payer bien cher la manie de toucher à tout",

«La colère ne peut que rendre nos efforts inutiles, c'est une mauvaise conseillère :

si on l'écoute, on a presque toujours à s'en repentir ",

«Le gourmand, dit un vieux proverbe, creuse sa fosse avec ses dents » (PIERRE, A., MINET A., MARTIN, A., Mlle, 1920).

16 Ces préceptes parsèment le manuel de morale: Nos petits amis, en 1920. On y voit comment le discours moral prescrit et proscrit un certain nombre de comportements, comment il cherche, par le verbe, à travailler les corps, à les fabriquer, par une mémorisation renvoyant à des préceptes simples ou à de petites paraboles. Il tente implicitement ou explicitement d'inscrire du verbe dans la chair, des mots dans les choses.

17 Vingt ans plus tôt, Marie PAPE-CARPANTIER - à l'origine de la création des Salles d'asile et des Jardins d'enfants -, et M. et Mme C. DELON, utilisent de petits fabliaux personnalisés, identificatoires, et cherchent à capter l'attention des enfants par des scènes prosaïques :

« La Colère. Quel tapage on entend dans la chambre ! - C'est le petit Armand qui est en colère. Il a brisé tous ses jouets, renversé une chaise. Il pleure, il crie, il rage... Il frappe de ses pieds, et ferme ses poings. Il a les yeux rouges, et toute la figure aussi ; ses cheveux tombent en désordre, tous ses traits font une affreuse grimace. Sa mère vient le prendre doucement ; elle le conduit devant une glace. Armand se voit, il se trouve laid et reprend son calme » (PAPE-CARPANTIER, DELON, C., 1900, 23). 
Même si le procédé didactique est un peu différent (l'identification à l'enfant et l'amour de la mère), de celui employé pour les maximes (où il s'agit de mémoriser par répétition quotidienne par exemple), le fond reste identique. Et c'est ce qui frappe lorsque l'on parcourt les manuels de morale, de la fin du XIXe siècle au début des années soixante, date de leur extinction : une stabilité des thèmes, leur récurrence, quel que soit le niveau de classe et le type d'enseignement, quelle que soit l'école (publique).

Ces thèmes, pour ce qui concerne les contraintes corporelles, sont :

- La préservation du corps (suicide, mutilations diverses),

- La lutte contre la paresse, la gourmandise, la colère, l'alcoolisme et l'ivrognerie ou, à l'inverse :

- La valorisation de la propreté, de la prudence, du travail, du courage, de la tempérance, de la sobriété et de l'exercice physique (le sport à partir de 1950 environ).

19 Tous ces thèmes définissent non seulement des préceptes concernant le comportement des élèves, mais aussi, pour le dire comme Michel foucault (1969), des systèmes de micro-pouvoir (voire de biopouvoir), une technologie de soi.

On est également frappé par la stabilité au cours du temps de certaines illustrations et paraboles : Le Laboureur et ses enfants est repris comme une antienne, Alexandre LE GRAND assassinant sous le coup de la colère et de l'alcool son meilleur ami, l'histoire du Chevalier D'ASSAS et du bataillon d'Auvergne ( «À moi Auvergne ce sont les ennemis »), les spartiates faisant absorber des quantités démesurées d'alcool à des Hilotes dans le but d'édifier leurs propres enfants sur les dangers de ce breuvage... Mais il est également impossible de compter les maximes qui sont reprises jusqu'à plus soif : « La colère est toujours mauvaise conseillère ", « la paresse est la mère de tous les vices ", « le gourmand creuse sa fosse avec ses dents »...

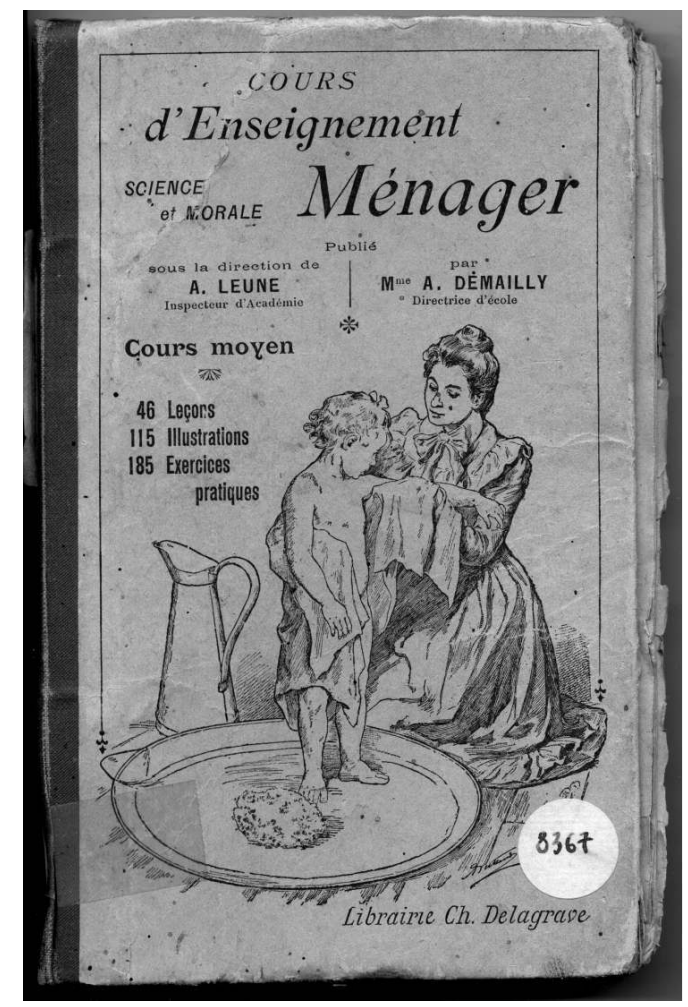

Illustration libre de droits : LEJEUNE A. et DEMAILlY A., Cours d'enseignement ménager, Science et Morale, Paris, DELAGRAVE, 1902. 
trail proposé analyse un corpus qui est particulièrement significatif lorsqu'il s'agit de cette question du corps et de la morale, des systèmes de contrainte et de contrôle du corps. Le travail d'archive a été réalisé, au Centre d'étude et de recherche en histoire de l'éducation (CEDRHE) de l'IUFM de Montpellier ${ }^{1}$ où l'on dénombre 234 différents ouvrages de «morale", de « lecture courante et de morale» ou encore des manuels contenant le mot «morale » dans leur titre, touchant tous les niveaux de classes et tous les types d'école (Primaire, Primaire supérieur, Secondaire). Il ne sera bien sûr pas possible de tous les citer en bibliographie.

Le travail présenté ici explore différents thèmes présents dans les manuels de morale de 1880 à 1966 (derniers manuels figurant dans le fonds) et tente d'en déterminer leur niveau de laïcité. Par ailleurs, il s'efforce de mettre en contexte ces manuels notamment du point de vue des structures matérielles mais aussi quand ce sera possible de celui des politiques et des politiques institutionnelles. La thèse défendue étant que les manuels tout autant que d'autres grands livres (Bible, Coran, Torah...) contribuent par le système de contraintes corporelles (Michel FoucAult parlerait de « biopouvoir ») qu'ils véhiculent à la fabrication de la civilisation des mœurs ou pour le dire sous forme métaphorique à la « fabrication d'anges ». Il resterait toutefois à prouver que ce qui est va être ici décrit a eu une certaine efficacité dans le réel. L'article démontre qu'il est difficile au regard de cette analyse d'affirmer que c'est une morale laïque qui est présente dans les manuels au cours $\mathrm{du}$ XXe siècle.

\section{L'interdit du suicide et des mutilations}

Ce thème n'est pas présent dans tous les manuels. Les ouvrages destinés aux plus petits (salles d'asiles et jardins d'enfants, voire cours préparatoire) ne proposent pas cette rubrique. Ce thème se fait aussi plus rare à partir de 1923, c'est-à-dire à partir de la suppression des «devoirs envers Dieu » des programmes et un peu plus tard (1926) des manuels.

La thèse de DURKHEIM, publiée en 1897 (DURKHEIM, 1897), n'est sans doute pas sans influence sur la présence de ce thème, elle est très souvent citée à partir du début du siècle. Bien que dans les manuels antérieurs à la publication de la thèse, le chapitre existe et s'appuie sur d'autres travaux, voire simplement sur la morale religieuse. De toute façon, depuis le concile de BRAGA (561-563), se supprimer est totalement condamné par l'église catholique. Au IXe siècle, l'anathème est prononcé contre les suicidés qui n'ont plus droit à une sépulture religieuse et aux prières pour le salut de leur âme. Ce point de contrainte corporelle repose donc sur des fondements catholiques ou chrétiens bien qu'il existe dans d'autres religions. Cependant, contrairement à d'autres thèmes présents dans les manuels, il n'est pas un péché capital ou une valeur cardinale ou théologale.

Dans le corpus, le suicide glorieux est toléré, par exemple, celui du Chevalier D'ASSAS, criant: «À moi Auvergne ce sont les ennemis !", au détriment de sa vie, en temps de guerre et par souci des autres. À l'inverse, toutes les mutilations sont proscrites, en tant qu'élément de lâcheté. Ce thème sera d'autant plus présent que l'on approchera de la guerre de 1914-1918. Paradoxalement, au sortir de celle-ci, le thème est peu évoqué.

Dans un autre registre, la mort de SOCRATE ou de MALESHERBES, également considérées comme héroïques, sont assez fréquemment citées, pour les plus grands. 

«Bataillons scolaires » et de la revanche contre les prussiens, anticipe les comportements dits « lâches".

"On racontait dans le village [...] qu'un mauvais sujet qui voulait échapper à la conscription s'était coupé deux doigts, [...]. Ce malheureux avait été mis en prison, ce qui était autrement désagréable que d'aller au régiment. Le maître fit comprendre aux élèves tout ce qu'il y avait de mauvais dans un pareil acte. [...] - Il y a des hommes, ajouta le maître, qu'une sorte de folie entraîne encore plus loin encore, jusqu'au suicide. Trop faibles [...] ils se dérobent par la fuite, par la mort volontaire, à l'accomplissement de leur devoir. [...] Je ne crains pas que vous en veniez jamais à une telle extrémité. Le suicide n'est que le dénouement fatal d'une vie criminelle, la faute suprême qui clôt une longue série de fautes » (COMPAYRE, 1883, 120).

Mais, même lorsqu'il s'agit de manuels pour jeunes filles, le suicide ou les mutilations sont considérés comme amoraux. En 1887, un manuel, conforme aux mêmes programmes, et destiné aux cours de 3e année de l'enseignement secondaire de jeunes filles, décrit: «l'immoralité du suicide; ses mobiles, ses conséquences » (HAUTIÈRE, 1887, p. 217).

Même dans les cas extrêmes (cancer, maladie incurable), dans de multiples manuels, la souffrance est conseillée plutôt que le suicide. Souffrir, plutôt que se suicider, est en effet considéré comme un geste d'amour pour les autres. On cite aussi, souvent, Jean-Jacques ROUSSEAU dans ce domaine : « La vie est un combat : honte à qui le fuit ! ", « Le suicide est un vol fait au genre humain (Jean-Jacques ROUSSEAU)» (NONUS, 1890, p. 50).

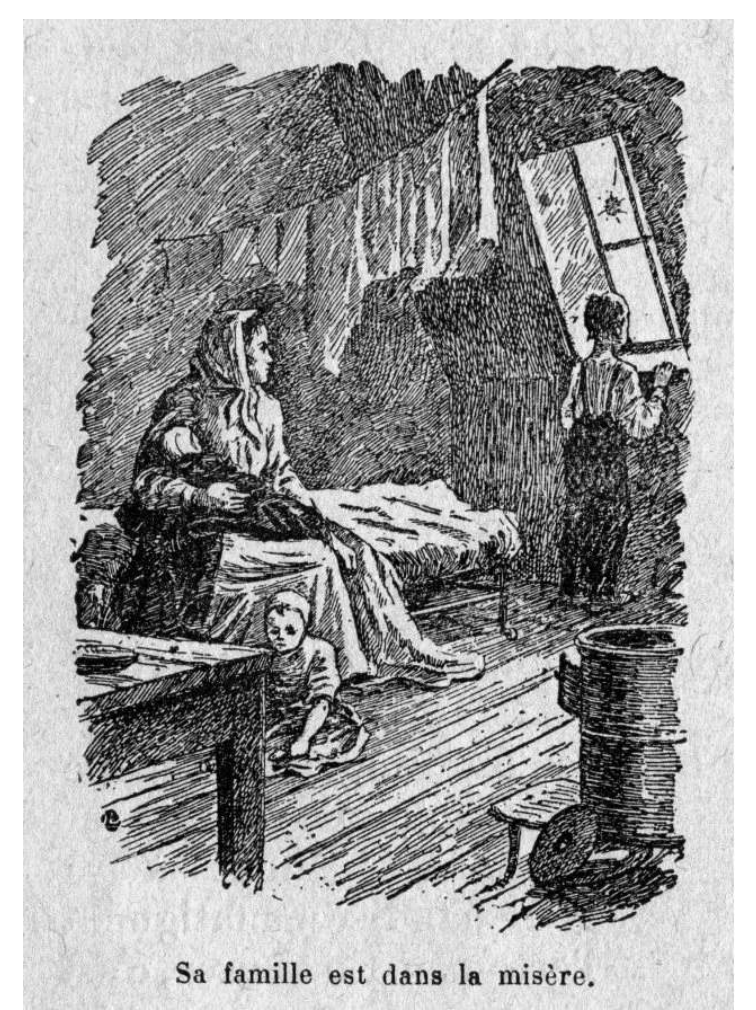

Illustration libre de droits : Illustration BREMOND E. et MOUSTIER D. L'éducation morale et civique à l'école, Paris, DELALAIN, 1920, p. 121.

La misère n'est pas non plus considérée comme une raison valable pour renoncer à l'existence. Dans un ouvrage de 1911, le suicide de GENOUILLET (pauvre), rédigé sous forme 
de parabole, est donné en exemple. Il y est décrit comme ne pouvant qu'accroître encore le désarroi et les difficultés de la famille, leur misère.

On doit souligner que les paraboles proposant des exemples négatifs décrivent toutes des familles pauvres, d'ouvriers, de paysans, d'employés ou de chômeurs. À l'inverse, les exemples moraux positifs mettent en scène des bourgeois (monsieur BOURGOIN), des aristocrates (le Chevalier D'ASSAS) ou parfois des personnages historiques ou prestigieux ( SOCRATE...), comme on l'a vu plus haut.

Sur ce même thème, plusieurs manuels, à partir de 1895, montrent l'augmentation du nombre des suicides en France, depuis 1843, à partir de statistiques établies par BRIÈRE DE BoIsmont (Du suicide et de la folie du suicide). Mais, dès 1918, ce sont les travaux d'Émile DURкнеiм qui sont les plus cités. Il s'agit ainsi de démontrer que la prospérité et le rayonnement militaire et économique de la France tiennent aussi à l'accroissement de sa population. C'est donc sur des bases apparemment rationalistes que le suicide est récusé et plus seulement sur des bases théologiques ou religieuses.

À partir de 1926, le thème du suicide est très généralement remplacé, dans les manuels, par l'hygiène, le souci du corps, la préservation de la maladie par la toilette, par le courage, l'abnégation ou l'exercice physique. Cette question réapparaît en 1956 avec celle de l'euthanasie. Dans ce cas, l'euthanasie est récusée au plan moral. La vision ne s'est donc guère transformée au cours du temps.

On voit donc clairement ici que l'influence de la religion chrétienne est importante pour la définition des préceptes moraux concernant le suicide. Pour autant doit-on en conclure que cette morale n'est pas laïque?

\section{La propreté et l'hygiène}

29 La question est présente dans tous les manuels de morale. Déchéance morale et saleté sont mises en corrélation tout au long de la période étudiée. Il est certainement possible de relier cela au fait que dans la chrétienté, le baptême est réalisé également par des ablutions d'eau (purificatrices) et que celles-ci participent à nombre de rituels, religieux ou non. 


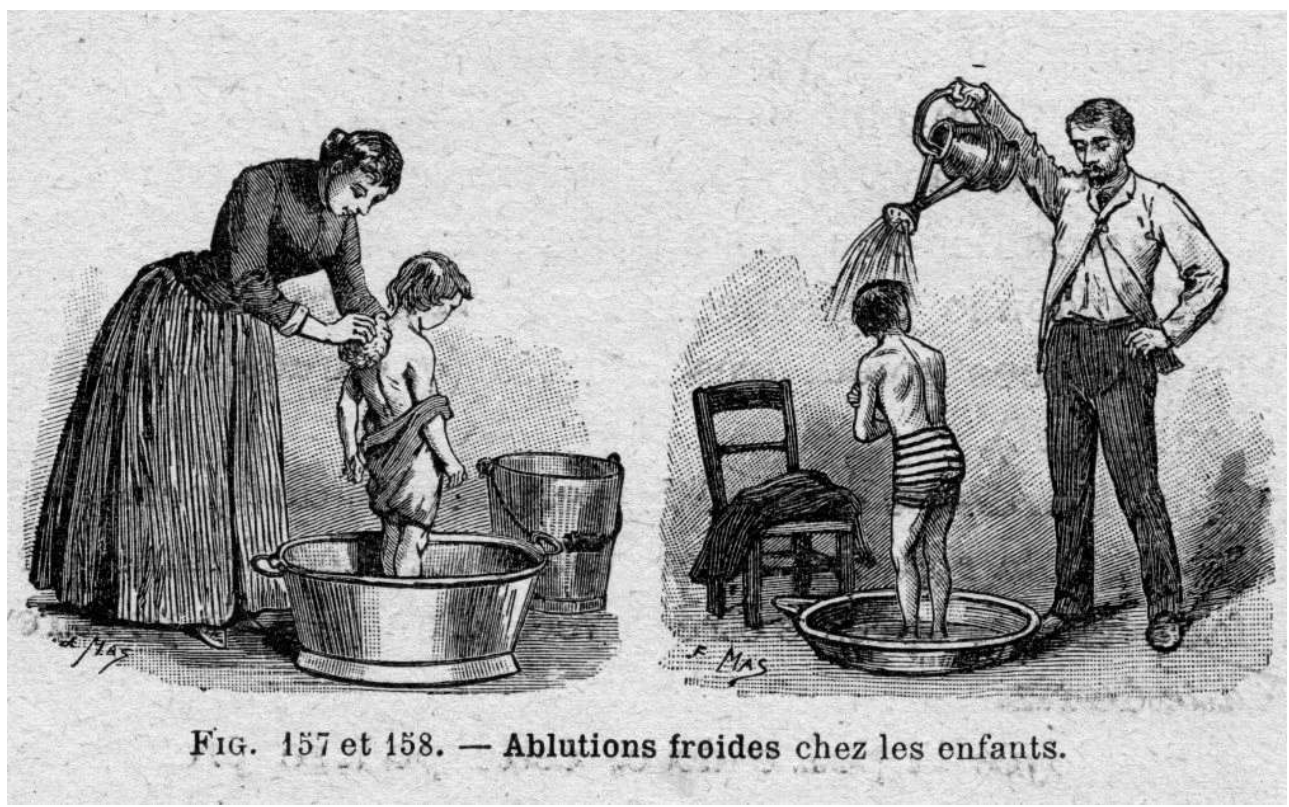

Illustration libre de droit

De ce point de vue, l'anthropologue Mary Douglas a bien perçu la dynamique associant ordre social et moral et souillure, voire pollution ou saleté (DOUGLAS, 1971, 1re éd. 1967). Pour elle, le corps est la métaphore de l'ordre social et, par voie de conséquence, de la morale.

Toutefois, lorsque l'on étudie les réalités matérielles, les adductions d'eau ou même, simplement, la présence des bains, on voit bien que cette contrainte est difficile à mettre en œuvre partout. Sans doute la bourgeoisie urbaine voire surtout parisienne peut accéder facilement à cette pratique, à la fin du XIXe siècle, mais, pour le peuple (rural), la massification de ces pratiques ne pourra pas avoir lieu avant les années soixante. C'est donc un modèle "bourgeois", de Civilisation des mœurs, qu'il s'agit de faire adopter aux élèves par le biais de ces manuels. En 1962, seuls 29 \% des foyers possèdent une baignoire ou une douche privée.

Par ailleurs, si l'eau a mauvaise presse (d'ailleurs, aussi, à boire) à la fin du XIXe siècle, c'est parce que les grandes pandémies de type choléra ou typhoïde sont encore très présentes. En 1893-94 une épidémie de choléra a décimé des milliers de personnes à Marseille, Toulon et Paris. Une deuxième touchera l'Europe aux environs de 1913. 


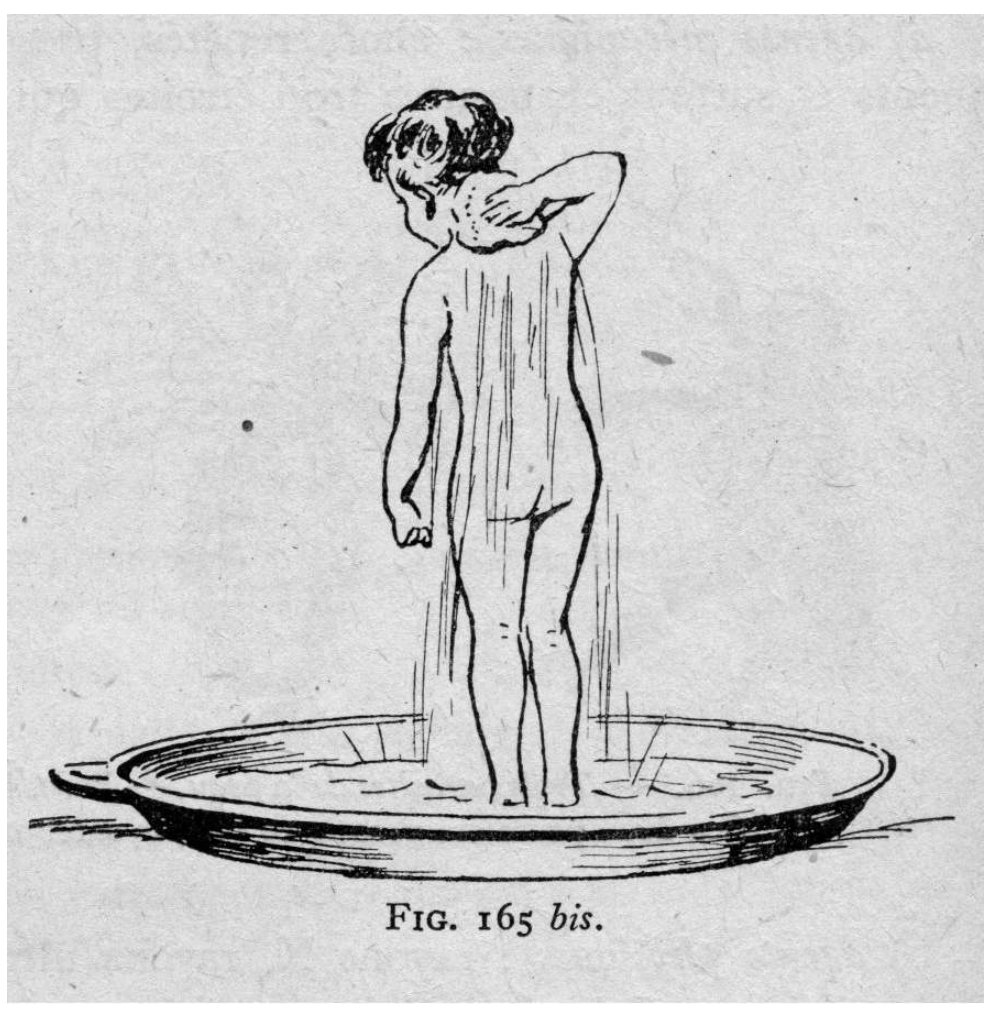

Reproduction libre de droit

31 L'ouvrage d'E. PECAUT est l'un des tout premiers à formuler des conseils précis dans ce domaine. Il définit la périodicité d'un bain complet par semaine.

« Nous diviserons, [...] ces ablutions en deux catégories, [...] nous dirons d'abord quelques mots de la toilette quotidienne, puis nous aborderons l'étude des bains.

L'habitude de se laver chaque matin le visage et les mains est suffisamment générale pour que sur ce point, l'hygiéniste n'ait pas grand'chose à redire. - Encore faut-il remarquer que beaucoup de gens, surtout beaucoup d'enfants, la pratiquent d'une manière trop sommaire.

Cette toilette matinale doit être faite, à grande eau et toujours à l'eau froide, hors les cas d'engelures ou de rhumes rebelles. - Il est bon de donner, dès le plus jeune âge, aux enfants, le pli de se laver en même temps le cou, la poitrine, les bras. [...] Il convient d'ajouter au moins une fois par semaine le lavage de la tête, à l'eau froide si les cheveux sont courts, à l'eau tiède s'ils sont longs » (PECAUT, 1882, p. 100).

Ce discours reviendra comme une antienne jusqu'en 1950 environ.

Toutes les techniques didactiques sont utilisées dans cette perspective. Pour les plus petits, on utilise des histoires très personnalisées et identificatoires ; pour les plus grands, ce sont les paraboles ou les aphorismes qui associent des auteurs célèbres et reprennent la logique de la propreté comme devoir social et devoir vis-à-vis de la pureté de l'âme, au moins jusqu'aux années vingt.

«Cours élémentaire: 1 . La propreté sert à témoigner le respect qu'on a pour la société et pour soi-même (BACON).

[...] 2. Ce qu'est la pureté pour l'âme, la propreté l'est pour le corps (ÉPICTETE)» ( DESPOIS et LABENNE, 1919, p. 123).

Pour les plus petits, d'autres techniques peuvent être utilisées. Il s'agit notamment de personnaliser encore davantage le processus didactique afin d'impliquer le très jeune enfant : 
«Je connais un petit garçon gentil, studieux; et je l'aime beaucoup. Pourtant le pauvre enfant a un défaut horrible, un défaut si répugnant qu'il m'empêche de l'embrasser : il est malpropre.

Il a ses mains noires ; tache ses vêtements ; [...] sa figure, son cou et ses oreilles sont très souvent sales, ses cheveux sont en désordre et ça le rend laid!

Je crois pourtant qu'il se corrigera car il s'est aperçu que sa malpropreté fait de la peine à sa mère » (PAPE-CARPANTIER M., 1900, 20).

À partir de l'extrême fin des années quarante, les techniques didactiques changent considérablement en faisant passer la douche ou le bain non pas comme un devoir mais comme un plaisir. Le discours glisse également de l'injonction et du devoir à l'appropriation et à la personnalisation. Ce n'est plus « on doit » qui est utilisé, mais « je ». En définitive, on passe ainsi de la morale à l'éthique.

«I. "J'éprouve un grand plaisir à être très propre." Chaque jour je prends soin de mon visage, de mes mains, de mes dents, de mes cheveux. [...] je prends des bains assez souvent. La malpropreté m'inspire de la répugnance » (BOURCEAU et FABRY, 1949, p. 200).

On peut même donner en exemple des textes très hédonistes, à la limite de l'érotisme comme celui de COLETTE :

«Aujourd'hui c'est [...] le jour où je prends ma douche, [...] Je me déshabille en vitesse et je tourne le robinet. [...] Enfin ça y est, voilà la douche réglée. Alors quel plaisir de sentir cette pluie fine qui s'abat sur moi et dont les mille petites aiguilles me picotent la peau! L'eau délicieusement tiède ruisselle le long de ma colonne vertébrale. Plaisir aussi de savonner vigoureusement avec un savon délicatement parfumé. [...] L'eau m'éclabousse quand je me rince, elle rentre dans mes yeux, dans mes oreilles, et je ris toute seule. Je ferme le robinet et je me secoue pour faire tomber les mille gouttelettes qui brillent sur ma peau comme des perles. Enfin, je m'essuie soigneusement. Je me sens bien, j'ai envie de chanter. C'est avec regret que je me rhabille.» (VILLARD, 1964, p. 6).

Le domaine de la propreté permet, en tout état de cause, de percevoir le processus d'appropriation de la morale, présent dans les discours, mais aussi sa déclinaison selon les niveaux de classe et les périodes. En définitive, on passe d'ablutions purification de l'âme, à une toilette devoir social, puis désir et plaisir individuel. Ainsi, peut-être, au cours du temps, la contrainte hygiénique se laïcise.

\section{La sobriété}

Au début de la période étudiée la sobriété n'a pas la même connotation qu'aujourd'hui. Elle touche davantage à l'abus de nourriture et est une déclinaison de la tempérance. Voici un exemple de cette connotation :

« L'ENFANT SOBRE

L'enfant sobre mange peu et selon son appétit et aux heures des repas seulement. Celui qui mange en dehors des repas, ou préfère des bonbons et des gâteaux à la nourriture, se charge l'estomac, [...] Enfants, soyez sobres ; car la sobriété conserve la santé de l'esprit et du corps » (NOEL, 1890, p. 132).

Les mêmes procédés que pour le chapitre précédent sont utilisés, parabole, histoire impliquant les enfants, contes... Une maxime revient aussi de manière récurrente (telle celle de D’ASSAS) :

«Il faut manger pour vivre et non vivre pour manger » (CAZES, 1890, 53). 
Il convient de rapporter cette question aux conditions de vie de la population française, au cours de la période. On se rend alors bien vite compte que les ouvriers et journaliers, les moins bien payés, n'ont pas, de facto, à mettre en œuvre ces préceptes. Bien au contraire, au moins jusqu'aux années vingt, bon nombre souffrent davantage de disette que d'intempérance. Dans ce cas, on comprend que les exemples de vertu (ne pas trop se nourrir) soient davantage illustrés par des personnages cossus. La "gourmandise", concept sur lequel on reviendra un peu plus loin, est, au sens plus extensif de gloutonnerie, l'un des péchés capitaux. À l'inverse, la tempérance est une vertu cardinale. Il est logique que ces éléments trouvent place dans les manuels de morale jusqu'aux années trente.

À partir des années cinquante, le discours bascule, comme précédemment, du " on doit " au « je », montrant cette intériorisation de la morale et ce passage d'une société morale (collective) à une société éthique, mais aussi probablement à l'individualisme contemporain :

«J'userai modérément des bonbons, gâteaux, friandises et sucreries dont l'abus est nuisible à la santé. Quand j'aurai quelques sous, je préfèrerai donner du pain aux indigents que de les gaspiller » (BOURCEAU et FABRI, 1949, p. 209).

\section{La tempérance dans le boire et le manger}

La tempérance est davantage associée à la question du boire que du manger. Il faut aussi préciser qu'existe toujours dans les manuels de morale un autre chapitre touchant à l'ivrognerie ainsi, pour les plus petits, qu'à la gourmandise. Pour les plus petites classes, généralement, les chapitres consacrés à ce dernier "défaut» sont nombreux. Mais la tempérance est une vertu cardinale chrétienne et surtout catholique.

Des statistiques, à différents moments de la période étudiée, mais plus particulièrement dans l'entre-deux-guerres, sont appelées à la rescousse pour démontrer, rationnellement, que les Français sont les mauvais élèves de l'Europe concernant les boissons alcoolisées. On y reviendra au chapitre sur l'alcoolisme. La question posée ici n'est pas celle de l'ivrognerie au sens strict mais celle de la tempérance en toutes choses. Autrement dit, il ne s'agit pas seulement de proscrire l'abus de boissons mais plus généralement l'intempérance pouvant conduire à des fautes plus graves.

Voici reproduit le type de visions véhiculées dans l'ensemble des manuels au cours de la période étudiée : 


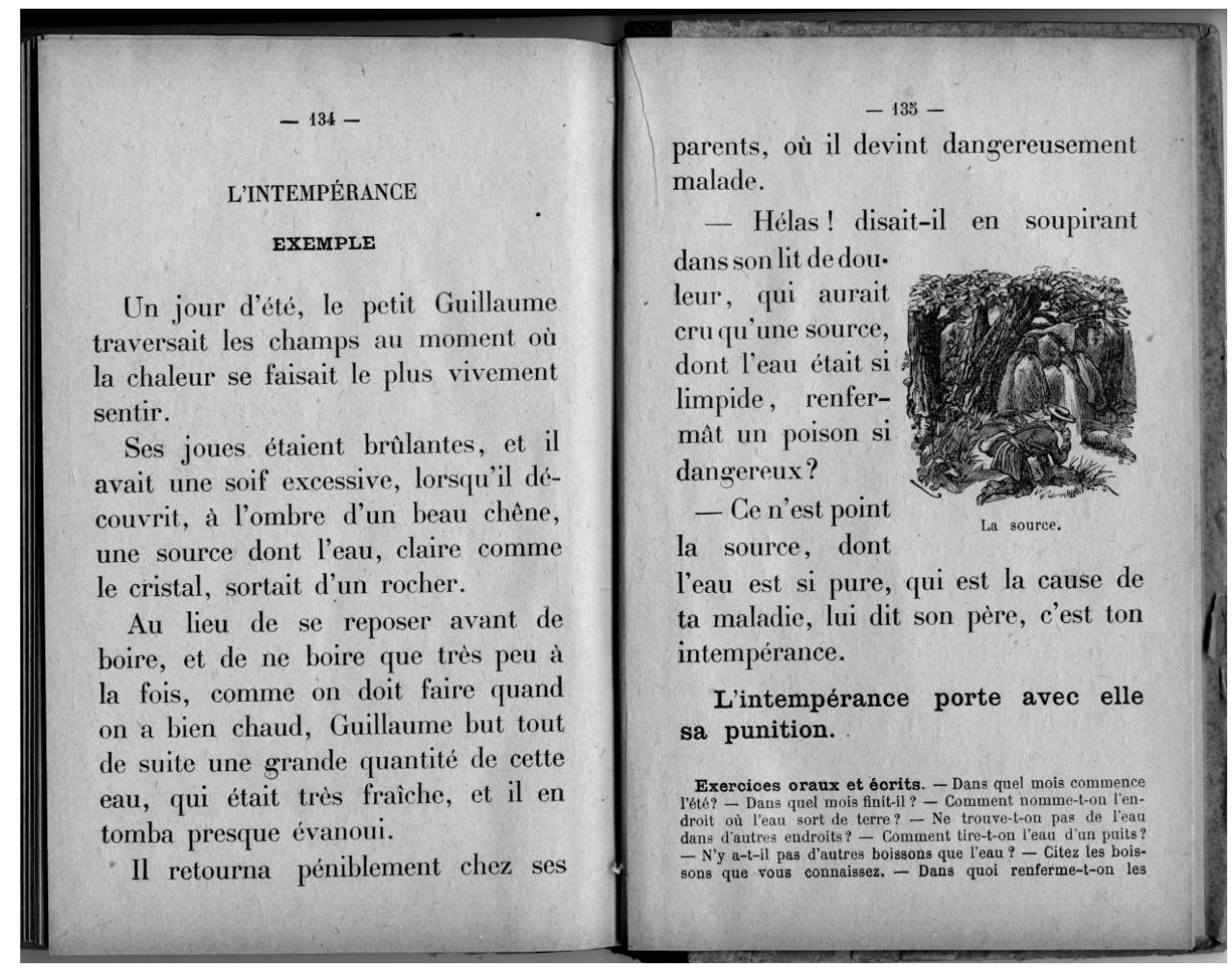

Reproduction libre de droit : CUIR A. F., Les petits écoliers. Lectures morales sur les qualités et les défauts des enfants, Paris, HACHETTE, 1901, p. 134-135.

Ce manuel est destiné aux plus jeunes, il ne parle donc pas de boissons alcoolisées mais seulement de la question du trop boire.

D'autres manuels sont plus explicites sur la question de l'abus de vin notamment:

« La tempérance n'est pas seulement une vertu du corps, qui interdit tous les excès de la gourmandise, les hontes de l'ivrognerie ; c'est aussi une vertu de l'âme, [...] qui épure nos penchants.

L'ivrogne est le pire des intempérants; mais, le fanatique est, lui aussi, un intempérant à sa manière [...]. À la tempérance, on peut rattacher plusieurs vertus : la modération dans les désirs, la modestie, l'amour de l'ordre [...] l'économie [...]» ( COMPAYRE, 1883, p. 126).

Un positionnement ascétique catholique est valorisé dans tous les domaines. Ce comportement est d'ailleurs habituellement attribué à l'éthique protestante, davantage qu'aux valeurs républicaines ou catholiques, même si, on l'a vu, la tempérance est, pour le catholique, une vertu cardinale et la gloutonnerie un péché capital.

Pour le reste, le système rhétorique et didactique fonctionne de la même manière qu'aux autres chapitres. On peut aussi trouver une formule, innovante, celle des bonnes résolutions :

\section{« RÉSOLUTION}

Je prends la résolution d'être tempérant, maître de tous mes instincts. Je serai sobre [...] Je n'accorderai à mes distractions que le temps qu'il est légitime de leur donner. Je ne me laisserai pas aveugler par mes affections et m'efforcerai de voir [...] où est mon devoir » (MAILLET, 1928, p. 161).

On voit là s'installer toute une technologie de soi, de micro-pouvoir (FOUCAULT, 1984) qui, si elle a une certaine efficace - ce qui reste à démontrer -, agit comme la constitution d'un processus de civilisation (un surmoi social), faisant passer en quelque sorte de 
l'animalité à l'humanité, de la chair au verbe, de la bête à l'ange.

Étonnamment, ces ouvrages, "républicains» pour la plupart, inculquent une morale chrétienne, religieuse, la plupart du temps catholique et non laïque. Mais existe-t-il vraiment une morale laïque?

\section{L'ivrognerie, l'alcoolisme, l'absinthe}

$\mathrm{Au}$ début de la période, les manuels ne confondent pas boire trop et être ivrogne ou alcoolique. En effet, l'alcoolisme ou l'ivrognerie ne sont pas associés au vin, à la bière, au cidre ou au poiré, décrits comme pouvant être bus raisonnablement (jusqu'à un demi-litre de vin par jour, voire un litre), mais surtout à l'absinthe, considérée comme démoniaque, jusqu'à son interdiction totale le 16 Mars 1915, en France, suite à des manifestations massives et une pétition, sous l'action des ligues de vertu (proches de l'église catholique et de l'ultra droite, notamment de l'Action française).

«Le plus connu, et le plus meurtrier, des apéritifs est l'absinthe, véritable poison lent, qui tue ceux qui en usent journellement, en les réduisant au plus misérable état (épilepsie, manie furieuse) ; l'effet de cette terrible liqueur ne s'épuise pas sur le malheureux qui en meurt; ses enfants, dès leur naissance, sont marqués du triste sceau de l'idiotie et peuvent hériter plus tard de l'épilepsie paternelle » (PECAUT, 1882, p. 42-43).

Longtemps après cette interdiction, les manuels en signaleront encore la supposée dangerosité. L'argument utilisé ici par Élie PECAUT est repris comme une antienne: le buveur transmet, de manière héréditaire, sa tare à ses enfants. Il est même précisé dans un manuel de 1904 que c'est un fait scientifiquement établi.

La condamnation de l'ivrognerie prend place dès le cours élémentaire. Les manuels, qu'ils s'adressent aux filles ou aux garçons, rejettent ce « ravalement au niveau de l'animal ».

«L'ivrognerie est la plus fatale de toutes les gourmandises. Une foule d'individus périssent pendant l'ivresse par suite d'accidents ; [l'ivrogne] descend au rang de la brute ; il ne reconnaît même plus ses proches ; il est querelleur, brutal, insolent.

Tout dans l'ivrogne inspire le dégoût. » (Nö̈L, 1890, p. 136).

Et dans ce domaine on retrouve aussi quelques sentences récurrentes. Par exemple: "l'ivrogne boit le sang de ses enfants ». On cite aussi très régulièrement l'histoire des Spartiates nobles faisant voir à leurs enfants des esclaves ivres afin de les dégoûter de la boisson. 


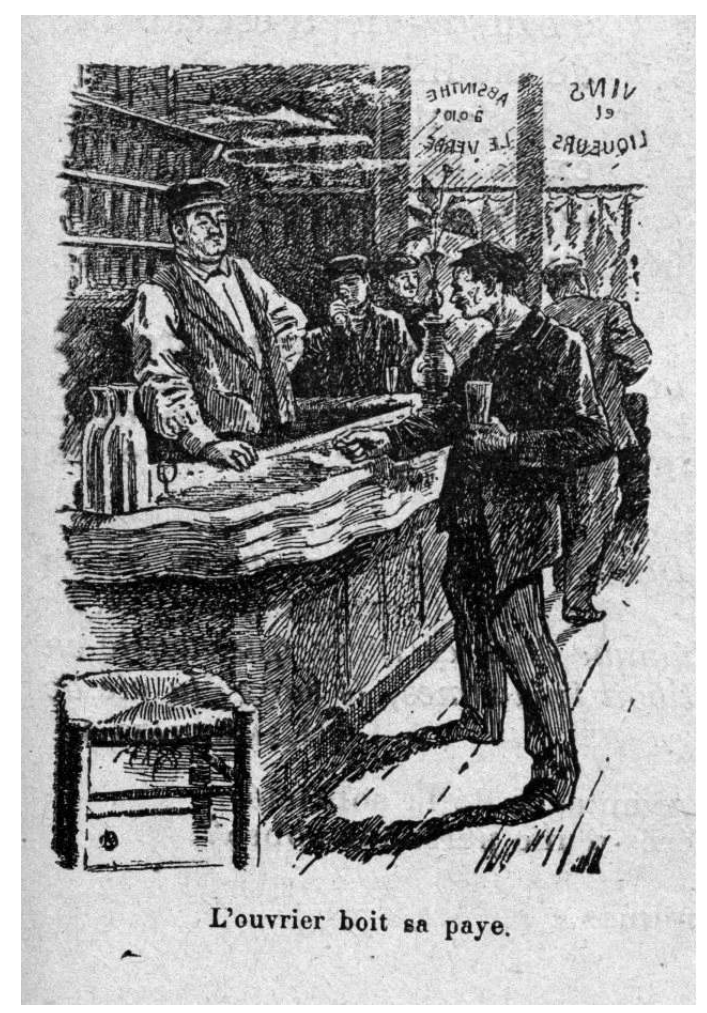

Libre de droits : Illustration BREMOND, E. et MOUSTIER, D. L'éducation morale et civique à l'école, Paris, DELALAIN, 1920, p. 119.

Un autre texte revient régulièrement qui explique, par l'abus de boisson, l'assassinat commis par Alexandre LE GRAND sur la personne de l'un de ses meilleurs amis.

Le passage progressif de l'injonction à l'appropriation individuelle du système de contrainte est également présent.

Mais, dans tous les cas, les exemples donnés concernent presque toujours les prolétaires voire, le lumpenprolétariat. Les ivrognes ne sont jamais des bourgeois ou aristocrates.

D'ailleurs lorsque l'on observe les doses proposées, même à la fin de la Seconde Guerre mondiale, on peut constater qu'elles ne sont pas identiques en fonction du type de travail :

«Un travailleur de force peut boire par jour 1 litre de vin à 10 ou $12^{\circ}$ mais un homme sédentaire n'en doit boire, au grand maximum, qu'un demi-litre. Aux enfants on en donnera peu et toujours coupé d'eau. Le vin mêlé aux aliments a moins d'action; il est recommandé de ne pas en boire entre les repas » (FoulonLEFRANC, 1944, p. 44).

Ce thème devient moins présent à partir des années soixante, c'est-à-dire dans les derniers manuels étudiés.

On doit enfin souligner que le système d'interdit de l'alcool s'appuie sur la même question que le chapitre précédent : le passage de l'humain à la bête, voire au démoniaque (l'alcool est décrit dans des paraboles comme le fils de la mort). On décrit une sorte de logique ou d'engrenage infernal qui va de la consommation d'absinthe, ou d'alcool fort, à la déchéance, au vol, au non-souci de la famille, de la patrie et au meurtre. L'ivrognerie est donc proscrite pour la séquence délétère qu'elle enclenche. Mais, encore une fois, il n'est pas certain du tout, comme on peut le voir, que ce soit sur une base laïque que repose la morale des manuels de l'école républicaine. Il s'agit plutôt d'une base religieuse catholique. 


\section{La gourmandise}

51 Pour la gourmandise, surtout présente dans les manuels adressés aux plus petits, c'est le même algorithme qui est réprouvé. Devenir dépendant des sucreries et autres douceurs donne une probabilité importante d'entrer dans un cercle vicieux.

Par ailleurs, la gourmandise est un péché capital dans la religion catholique. On comprend donc, qu'au début du siècle et au moins jusqu'en 1923, ce soit la morale chrétienne et surtout catholique qui conduise à en indiquer l'interdit fondamental.

Mais, plus tard, la gourmandise est réprouvée pour les «mauvaises actions » qu'elle peut engendrer (vol) mais aussi à cause du fait qu'elle va nuire nécessairement à l'état de santé du gourmand. Le gourmand est fréquemment décrit comme se punissant lui-même :

«L'enfant qui se laisse aller à la gourmandise s'inflige souvent à lui-même sa punition » (PIERRE, LETRAIT, BODIN, 1909, p. 65-66).

Par ailleurs, comme ces chapitres s'adressent aux plus jeunes, de très nombreux fabliaux personnalisés y trouvent place, comme «Le palais de Dame FRIANDE », où un enfant se trouve sollicité, presque jusqu'à l'agonie, à la consommation de sucreries, fort heureusement dans un rêve, revenant dans plusieurs ouvrages. L'histoire du roi " GOURMANDEAU ", finissant par mourir de sa gourmandise, elle aussi publiée dans plusieurs manuels. Un autre fabliau concernant un gourmand puni d'avoir ingurgité trop de «baba », revient de manière récurrente.

On retrouve aussi, à ce chapitre, les aphorismes déjà énoncés au chapitre sur la tempérance et la sobriété : «il faut manger pour vivre et non vivre pour manger » ou encore «le gourmand creuse sa tombe avec ses dents». On fait également appel ici à VOLTAIRE (ZADIG) ou à LA BRUYÈRE (GNATHON) pour édifier les lecteurs plus âgés.

Il faut pourtant souligner que les moyens des ouvriers et employés les plus défavorisés, au moins jusqu'aux années vingt, ne devaient pas leur permettre d'acheter beaucoup de sucreries, pas plus qu'ils ne devaient pouvoir se payer de multiples boissons alcoolisées au café (ils pouvaient abuser du vin). Or, c'est avant tout à eux que ces injonctions sont adressées et non à la bourgeoisie qui est toujours décrite comme vertueuse. En ce sens la "classe laborieuse» dans ces manuels est bien considérée comme la «classe dangereuse ». En effet, ces manuels sont très massivement adressés aux enfants du primaire et c'est pour eux que la grande majorité a été rédigée.

Par ailleurs, le processus de personnalisation est présent dans ce domaine comme dans les autres à partir des années cinquante. Ainsi, en 1949, la maxime suivante conclut la 37e leçon intitulée : « Il faut combattre la gourmandise »:

«J'userai modérément des bonbons, gâteaux, friandises et sucreries dont l'abus est nuisible à la santé. Quand j'aurais quelques sous, je préfèrerai donner du pain aux indigents que de les gaspiller en choses inutiles et parfois nuisibles. » (BOURCEAU, E. et FABRY, R., 1949, p. 209). 


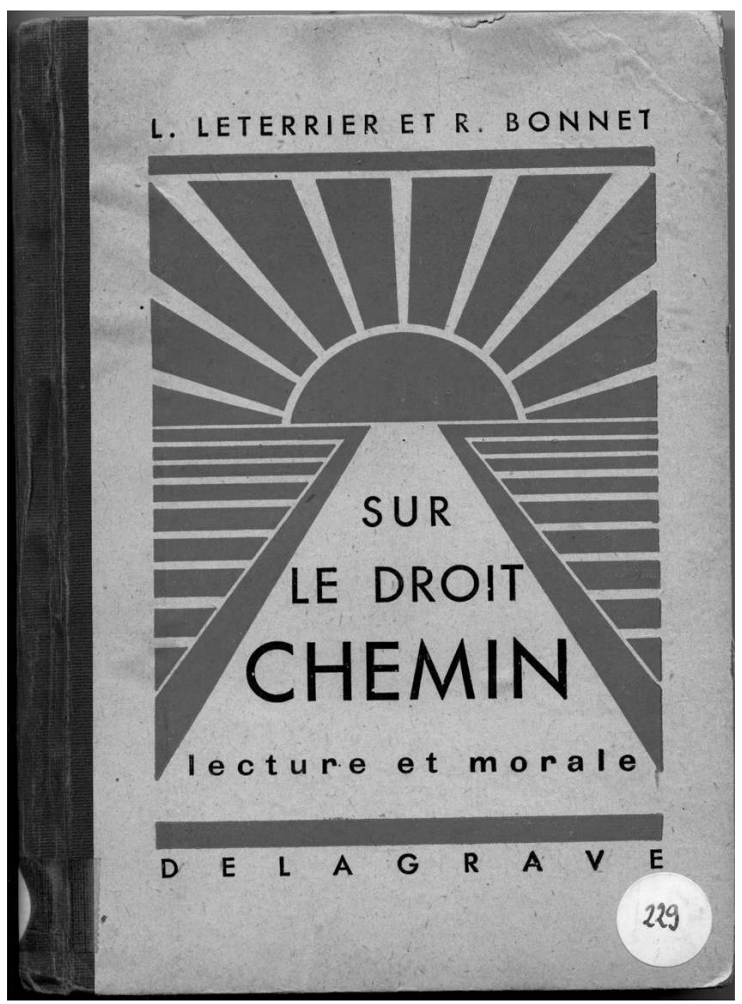

Libre de droits. LETTERIER L. et BONNET R., Sur le droit chemin. Lecture et morale, Paris, DELAGRAVE, 1940.

\section{gourmandise, mais bien la cascade inéluctable qu'elle peut entraîner, conduisant à des} «péchés » de plus en plus grands.

Enfin, on doit noter l'association: gourmand-honteux-laid dans de très nombreux passages. La honte (émotion) est associée à un " défaut » (la gourmandise) dans le but de produire une efficace. Une vertu (" avoir bon cœur », c'est-à-dire garder son argent pour le donner aux pauvres et non le dépenser en sucreries), proche de l'une des trois vertus théologales (l'amour du prochain, la charité) chrétiennes, est décrite, dans de nombreux passages consacrés à la gourmandise, comme remise en cause par ce vice ou défaut.

Pour terminer sur ce point, si le mot péché est utilisé à la fin du XIXe siècle, par la suite c'est, plus fréquemment, le mot "défaut", un peu comme si les auteurs de manuels souhaitaient se détacher de la vulgate chrétienne en prenant une distance avec le lexique utilisé par l'institution ecclésiastique. Pour autant, peut-on affirmer que la morale se laïcise?

\section{$X$. La colère vs la douceur}

Dès les tout premiers manuels de morale étudiés, la colère est posée comme une forme de folie qui fait passer de l'humain à la bête

"Comme l'a dit un ancien, la colère est une courte folie. Regardez l'homme en colère: son visage se contracte, ses yeux sortent de la tête, sa bouche a un mouvement convulsif. Il prononce des paroles qu'il ne prononcerait jamais s'il était de sang-froid, et qu'il regrettera d'avoir prononcées.

[...] Il est exposé dans l'accès de son comportement à commettre des actions 
irréparables, à insulter ceux qu'il aime le mieux et auxquels il doit le plus de

respect, ses amis, ses proches, ses parents » (MEZIÈRES, 1893, p. 84-85).

\section{civilisé et social acceptable, a fortiori, pour une femme. On verra cela plus loin.}

En tout état de cause, si la colère est, elle aussi, un "péché capital " pour la religion catholique, ce n'est encore une fois pas elle, en soi, qui est condamnée par les manuels de morale mais la cascade, l'algorithme fatal qu'elle peut engendrer :

« [l'homme en colère] ne sait plus ce qu'il fait. Sait-il si la colère ne l'emportera pas jusqu'au crime, s'il ne lèvera pas la main sur son père, sur sa mère, s'il ne portera pas quelque mauvais coup à un camarade innocent ? Beaucoup de meurtres ont été commis dans des accès de colère

[...] dans les transports de fureur immodérés, des amis ont tué des amis, des frères ont tué des frères, des fils ont même frappé leurs parents.

Les coupables se désespèrent quand le mal est fait. Ils se seraient épargnés bien des remords s'ils avaient commencé de bonne heure à résister aux tentations de la colère » (Idem, 85$)$.

Les ouvrages utilisent, ici, comme pour l'ivrognerie, l'exemple d'Alexandre LE GRAND tuant son ami.

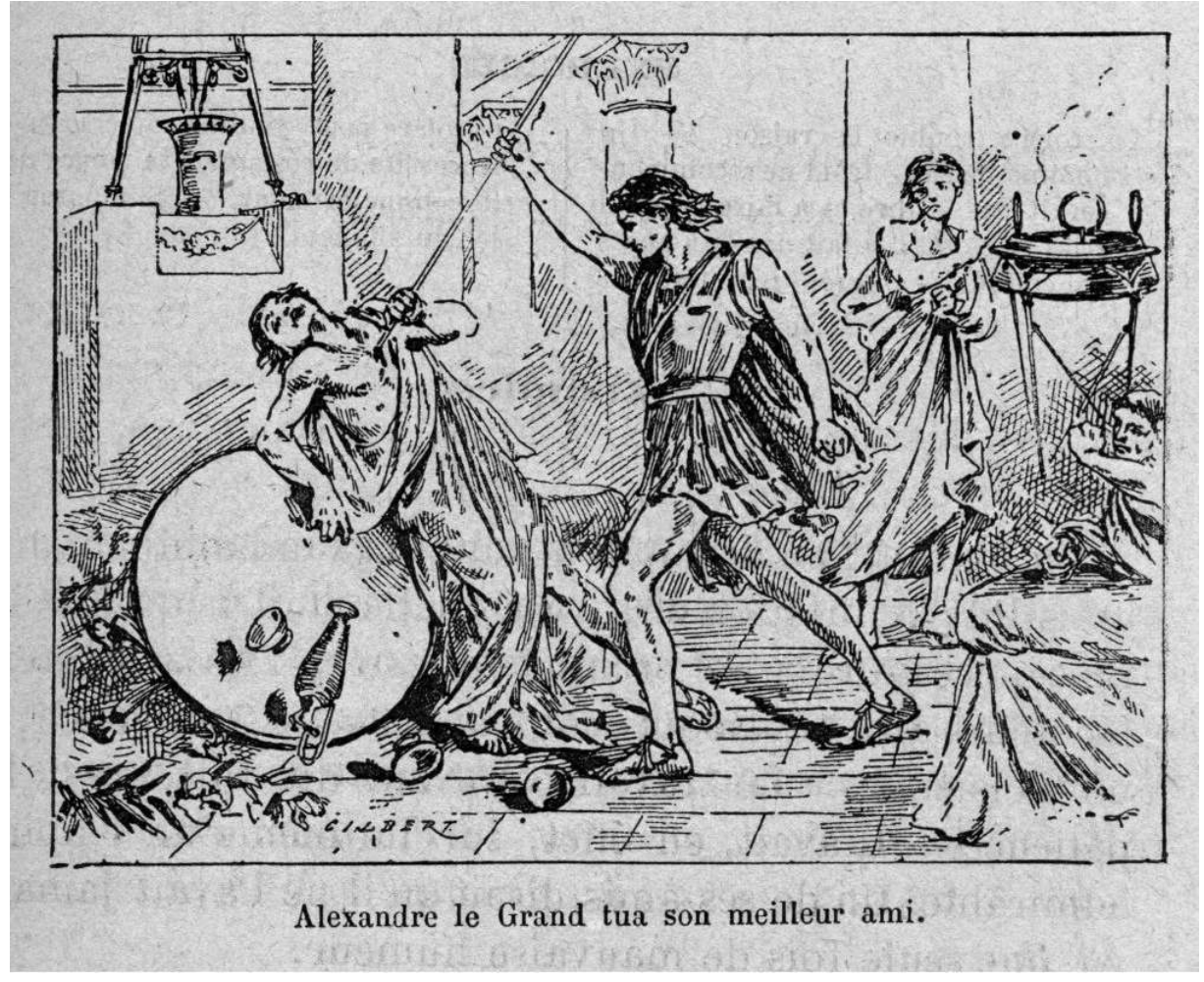

Libre de droits. Illustration MEZIÈRE M. A., Education morale et instruction civique, Paris, DELAGARAVE, 1893 , p. 87.

À partir du début du XXe siècle, on utilise des dessins au trait pour soutenir le discours et le renforcer. On en a vu de nombreux exemples en cours de texte. À partir du sortir de la Seconde Guerre mondiale, les photos sont de plus en plus nombreuses. Elles viennent pratiquement toujours en redondance du discours, comme pour lui donner plus de force. Pour le thème étudié, un certain nombre de comportements, particulièrement calmes sont associés à des Anglais célèbres. Le self control semble être considéré comme un comportement spécifique à cette «race » calme et non à la «race française », plus 
sanguine. Sont ainsi cités en exemple un Premier ministre Anglais ou une personnalité célèbre, M. HUMPDEN, membre du parlement dont le self control est particulièrement valorisé. Mais, là encore, à de rares exceptions près (Alexandre LE GRAND), ce sont toujours des «bourgeois» qui ont des comportements calmes, alors que les pauvres ont des comportements colériques.

61 Par ailleurs, la douceur est une valeur extrêmement valorisée lorsqu'il s'agit de femmes et de jeunes filles. Les manuels spécifiques aux filles insistent beaucoup sur cette valeur qui doit être, principalement, portée par elles. Une femme est presque toujours décrite, comme le régulateur de douceur du foyer. En 1895, un manuel intitule même un chapitre : "Nécessité de la douceur pour la femme» et ce chapitre se conclut par la maxime suivante, probablement à apprendre par cœur :

«Si la douceur n'est pas la première vertu de la femme, elle est peut-être son plus puissant moyen de bonheur » (SEIGNETTE, 1895, p. 59).

À l'inverse, le journalier, de préférence ivrogne ou intempérant, est vecteur de violence et de colère. Le meurtre, décrit comme en augmentation (contrairement à l'objectivité des statistiques), est le plus souvent associé à un accès de colère. C'est pour cela que le binaire, patient vs colérique est utilisé comme un système manichéen dans lequel le lecteur doit trouver sa propre réponse :

«L'homme colère :

Est insensé : perd la raison. Est faible : n'a pas la force de résister à une passion. N'est plus libre. Est injuste. Se dégrade. S'expose à commettre des actes qu'il regrettera par la suite.

L'homme patient :

Conserve sa pleine raison. A une grande force de caractère. A toute sa liberté. Juge

librement et équitablement. Respecte sa dignité. Agit en conscience. » (Idem, p. 139).

Et l'ouvrage cité ici renvoie, à ce point, au Tour de France par deux enfants de G. BRUNO ( alias Augustine fouilLÉE) et, notamment, au passage de la page 206 consacré, aussi, au binaire antagoniste: "Brusquerie et douceur». Deux maximes sont par ailleurs assez souvent utilisées concernant la douceur : "La douceur désarme les méchants » et «il n'est de plaie au cœur que la douceur ne ferme ».

Pour le reste, évidemment, aux plus petites classes sont adressés les fabliaux et les modèles identificatoires, aux plus grands les illustres prédécesseurs. Et, encore une fois, le processus de personnalisation devient évident au tournant des années cinquante. Il n'en reste pas moins que la colère est un péché pour la religion catholique et devient l'un des interdits fondamentaux de manuels que l'on imaginerait originellement laïques.

\section{La paresse et le travail}

«Gloire au travail ! Gloire à l'homme champêtre, Gloire à celui qui féconde le sol! Picard, Breton, Limousin, Cévenol, Gloire à celui qui mène les bœufs paître ! Gloire à l'homme champêtre!» (LAUney, H., LAUney, J. et RASCOL L., 1921, p. 135, par exemple),

64 C'est ainsi que s'achève le chapitre consacré au thème dans plusieurs manuels. Mais d'autres maximes sont présentes, par exemple: «Le travail enrichit, la paresse appauvrit ». La plupart des manuels comporte un chapitre sur ce sujet souvent intitulé : « Travail et paresse », mais parfois portant seulement sur la question du travail. 
65 À l'inverse, on connaît la maxime répétée à l'envi dans les manuels « la paresse est la mère de tous les vices ». La paresse est, en tout état de cause, elle aussi, un péché capital dans la religion catholique. Mais, dans le corpus, c'est moins elle qui est décriée que le travail qui est valorisé. C'est, en ce sens, une morale bourgeoise qui trouve place dans ces manuels et non une morale aristocratique.

Tous les procédés vus précédemment sont utilisés pour favoriser l'apprentissage de ce qui est décrit comme une loi de la nature, du moins au début du siècle; par la suite on trouvera plus fréquemment la phrase suivante : « Le travail est la loi humaine » (POIGNET et BERNAT, 1919, 32e leçon non paginée).

Par ailleurs, le travail manuel est très souvent mis en valeur, peut-être davantage que le travail intellectuel, au début du siècle notamment, ce qui est logique au regard du contexte socio-économique. La figure du « Menuisier BRISSOT » en témoigne. Celui-ci se dit ravi de son travail et il peut affirmer :

«Le travail [...], c'est ma santé. Le jour où mes bras ne pousseront plus la varlope, je ne mangerai plus avec le même appétit et je n'aurai plus la même vigueur » ( DEVINAT, E., 1920, 131).

67 Une fable de LA FONTAINE revient aussi comme un leitmotiv dans les chapitres consacrés à ce sujet: «Le laboureur et ses enfants». Comme on le sait, la fable commence par: « Travaillez prenez de la peine, c'est le fond qui manque le moins » et se termine par « De leur montrer avant sa mort que le travail est un trésor ». Bien entendu, pour les élèves de cours élémentaire ou de cours moyen il faut apprendre la fable par cœur.

Un ouvrage de 1920 parle, lui, purement et simplement de "sainteté du travail » ( BREMONT et MOUSTIER, 1920, 158). Cet ouvrage précise par ailleurs :

« Tout travail est utile qu'il soit intellectuel ou manuel, pourvu qu'il soit exécuté avec courage et intelligence. Il n'y a pas des travaux nobles et des travaux avilissants. Tous les travailleurs consciencieux ont droit au respect et à l'estime » (ibid., 161). Il s'agit, donc, davantage de valoriser le travail que de stigmatiser la paresse. On peut rappeler qu'existe, à cette époque et jusque dans les années soixante-dix, une médaille du travail remise à tout travailleur méritant. Sur un autre plan, des études de Suzane MOLLOT , dans ces mêmes années, montraient qu'à performance égale un élève travailleur (vs dilettante) était valorisé par les enseignants. L'église catholique et la religion chrétienne promeuvent également le travail - en condamnant la paresse et l'oisiveté - même s'il est aussi considéré comme un châtiment de Dieu infligé à l'Homme. 


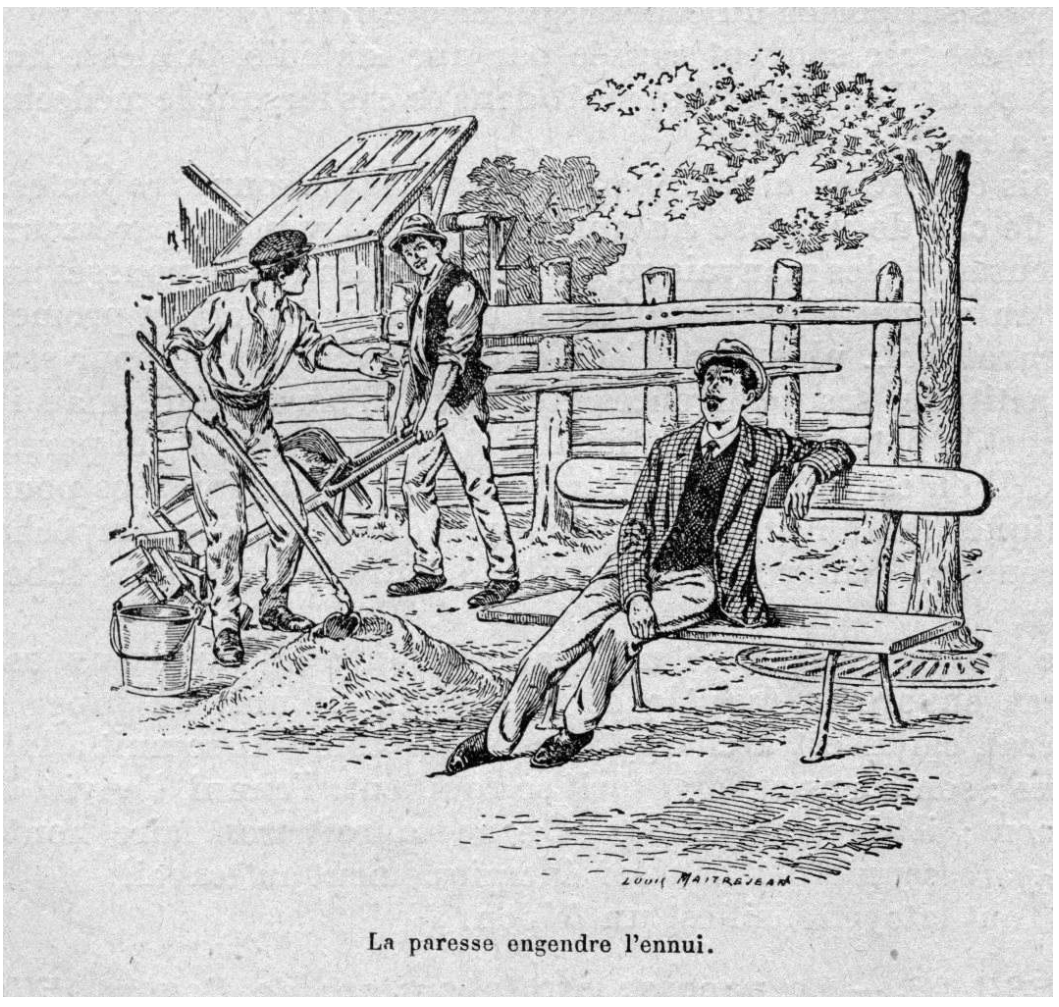

Illustration BREMOND E. et MOUSTIER D., L'Education morale et civique à l'école, Paris, DELALAIN, 1920, p. 164 : « La paresse engendre l'ennui. »

La maxime de Vichy : «Travail, famille, patrie », est certainement en phase avec la société de l'époque, même si elle sera réprouvée après la libération. Le travail, pour beaucoup de sociologues contemporains, est le centre de la société de 1880 à 1968 au moins.

Par contre, le loisir semble se substituer à lui selon d'autres chercheurs (DUMAZEDIER, 1988 ou DURAND et MERRIEN, 1991) à partir des années quatre-vingt, quatre-vingt-dix. Les manuels de morale du fonds étudié s'arrêtent en 1966. En conséquence, il est difficile, si ce n'est par la présence de plus en plus grande du travail intellectuel, de dire qu'il y a eu créativité dans ce domaine pour les manuels.

Par petites touches se construit donc un système de contrôle de plus en plus structuré, très proche de la vulgate chrétienne concernant les péchés capitaux et les vertus cardinales. Si l'on veut bien admettre que, s'adressant à des centaines de milliers d'enfants, voire des millions au cours du temps, il ait eu une certaine efficace, on peut mieux comprendre, grâce à cette étude, quel est le mode de civilisation contemporain en France.

\section{Le courage et la prudence}

71 La prudence est associée aussi à la tempérance. En ce sens, elle peut être une vertu cardinale. Mais, à l'inverse, le courage est lui aussi valorisé. Cependant, il y a une modification progressive des manuels sur ce point. $\mathrm{Si}$, au début de la période étudiée, être courageux c'est sacrifier sa vie pour la patrie ou pour la société, après la Seconde Guerre mondiale et même parfois dans l'entre-deux-guerres, être courageux, ce peut être aussi être prudent ou sauver les autres. 
72 Ainsi si le courage du Chevalier D'ASSAS est souligné par temps de guerre, on valorise également, pendant toute la fin du XIXe siècle et le début du XXe, le malade qui souffre en silence, le handicapé (on dit : mutilé, invalide ou estropié) qui se met au service des autres malgré ses difficultés.

73 À partir de la période des années trente, le courage est lié au travail, au garçon qui en sauve un autre de la noyade, au péril de sa vie. On trouve aussi courageux un enfant qui travaille dans des conditions difficiles. Mais la prudence est aussi associée au «vrai courage ». En effet, le courage irraisonné est à partir, au moins des années vingt, considéré comme néfaste car il peut être associé au suicide. La prudence consiste à être courageux mais sans mettre inutilement sa vie en jeu. La tempérance permet de départager le vrai du faux courage.

Voici ce que l'on peut trouver dans un manuel de 1920 :

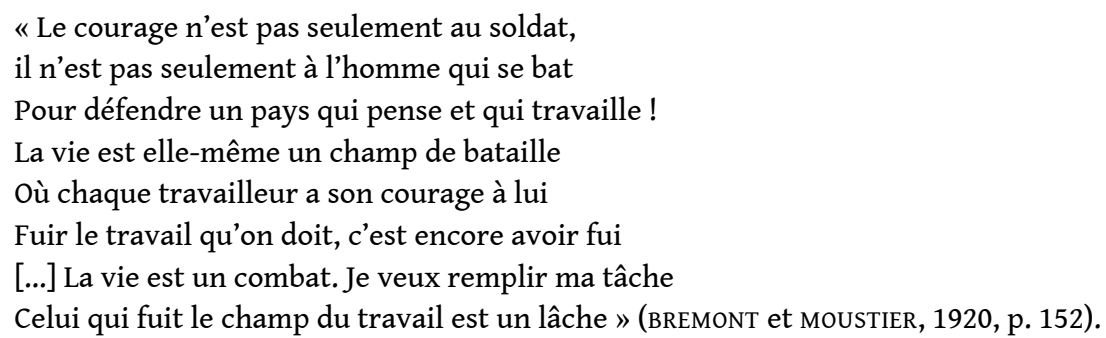

On voit ici l'association du chapitre précédent avec celui du courage, mais finalement d'un courage régulier et prudent, pourrait-on dire apollinien et non dionysiaque, participant davantage de la raison que de la pulsion et de la passion ou de l'émotion.

À partir des années quarante, le courage sera de plus en plus souvent associé au sport et à la possibilité d'aller au-delà de ses limites pour réussir un exploit sportif.

En fait si l'on continue à parler, bien sûr, de héros de guerre, après 1945, c'est de plus en plus la victoire des difficultés quotidiennes : vaincre la peur, la tentation, produire un effort physique ou intellectuel, travailler dur, qui est désignée comme acte de courage. On parle des héros de tous les jours.

De nombreuses techniques didactiques sont utilisées comme dans les autres domaines. On peut y retrouver les auteurs prestigieux, les hommes célèbres, les héros guerriers mais aussi des enfants comme modèles identificatoires :

«Des enfants jouent. L'un d'eux fait une chute, se blesse grièvement à la tête et perd connaissance. Effrayé à la vue du sang et de l'immobilité de l'enfant, ses camarades ont la faiblesse de prendre la fuite. Un seul a la présence d'esprit de laver la plaie du blessé, et, après l'avoir bandée avec son mouchoir, il aide à le transporter au domicile de ses parents, quand du secours arrive. Cet enfant a fait preuve de courage » (CURE, HOUZELLE, 1895, p. 141).

Ici le courage ce n'est pas un acte inconsidéré mais bien un acte posé, à la portée de tous.

On peut utiliser aussi, comme précédemment, la technique du binaire :

«L'homme courageux :

Conserve sa volonté et sa pleine raison. Ne désespère jamais. Surmonte les obstacles. Est surtout grand dans le malheur. Accomplit toujours son devoir.

L'homme peureux :

Sa volonté et sa raison sont annihilées. Prend une résignation passive et humiliante. Se laisse abattre par les difficultés. Perd son sang-froid dans le péril ou l'infortune. N'accomplit son devoir que lorsqu'il a peu de mérite à le faire » (ibidem). 


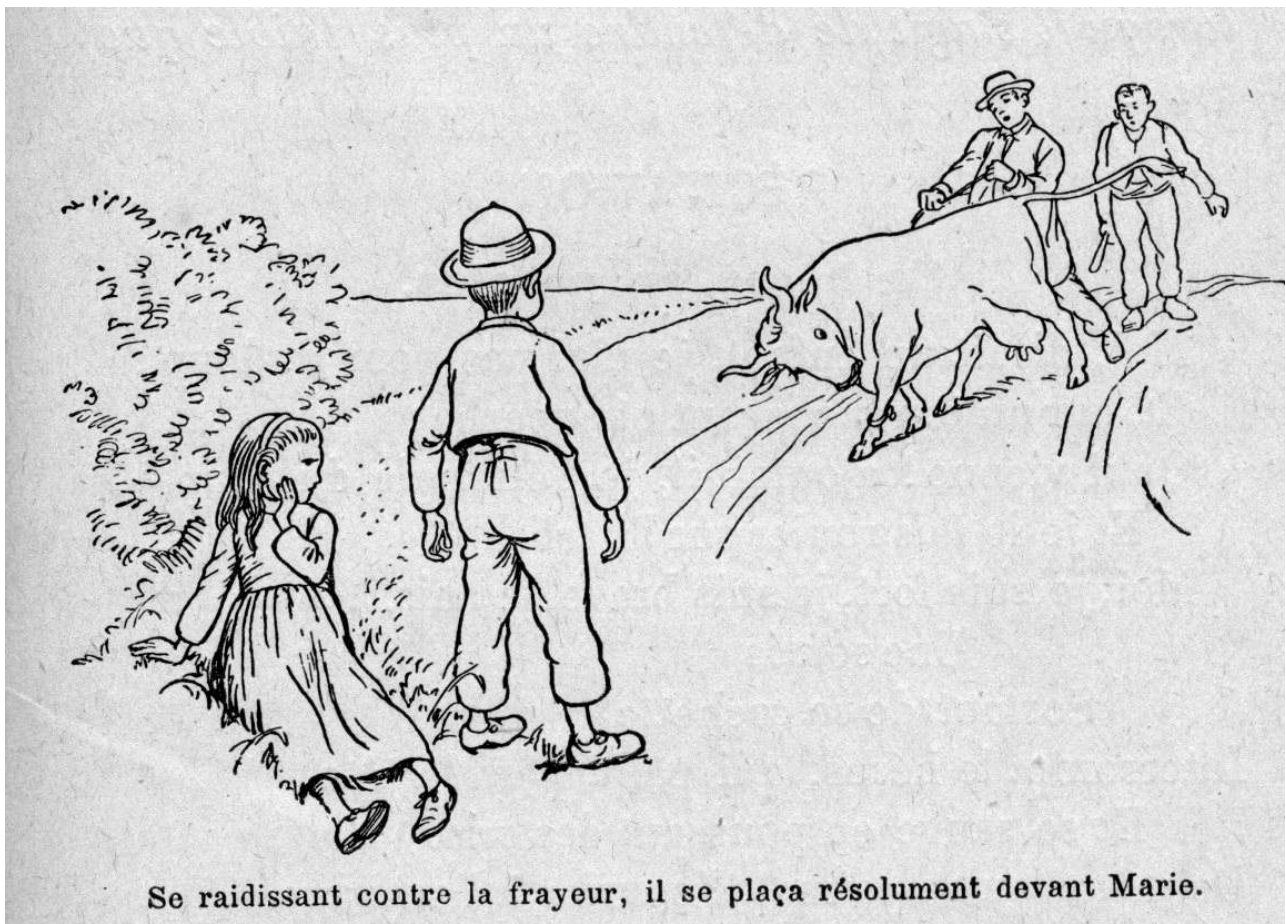

Illustration PIERRE A., LETRAIT Mlle, et BODIN Z., Lectures et récitations morales, Paris, NATHAN, 1909, p. 93.

Pour les manuels, il semble décisif de discriminer peureux, poltron et prudent. C'est ce que fait un manuel de 1901, en donnant l'exemple identificatoire de Lubin. La situation est la suivante : des enfants jouent à sauter par-dessus un trou de marne très profond. Lubin, seul, ne veut pas sauter, disant que l'on peut manquer son coup et tomber dans le trou. Un autre enfant le traite de poltron. Mais un enfant ayant mal jugé son élan, tombe et se blesse assez gravement (il se casse la jambe). À ce moment les enfants jugent Lubin prudent et non poltron.

La prudence peut être, ainsi, pour les rédacteurs de manuels, associée au courage. Ils ne valorisent pas un courage inconscient. En définitive c'est, dans une sorte d'oxymore, un courage prudent qui l'est ou du moins un courage raisonné et raisonnable.

\section{L'exercice physique, le sport}

L'exercice physique, n'est jamais présent dans les textes religieux : il n'est ni une vertu théologale, bien sûr, ni une vertu cardinale, ni d'ailleurs une pratique valorisée, il fait partie des plaisirs considérés comme sains, au même titre que la lecture, le dessin, la musique à l'inverse des plaisirs malsains, le jeu d'argent, le café, la boisson, le tabagisme, etc. 


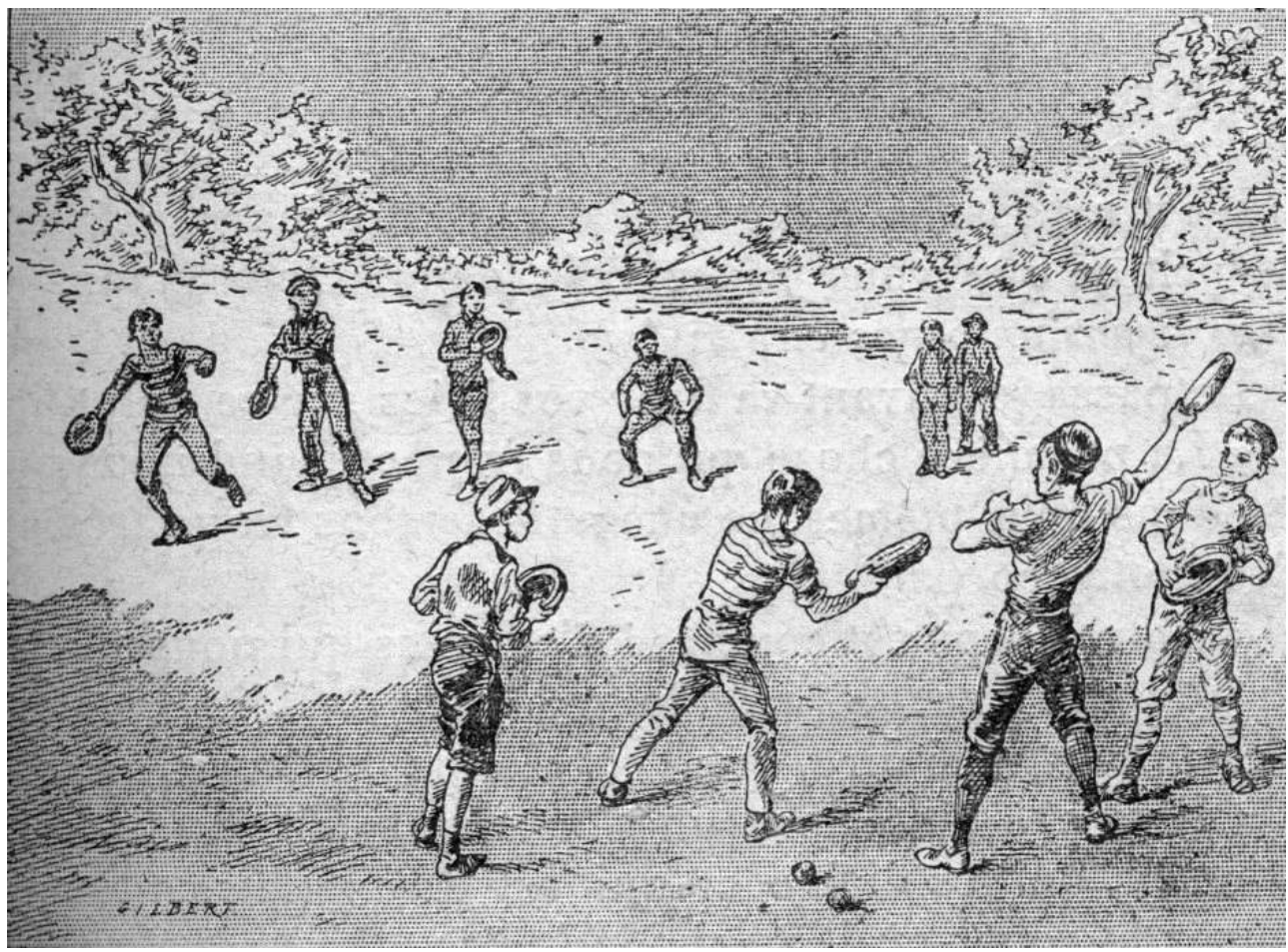

Illustration CAZES E., Instruction morale et civique, Paris, DELAGRAVE, 1890, p. 49. de lutter contre l'oisiveté et la paresse. Il est donc un analogon du travail dans le domaine du loisir. Tout ce qui a été dit au chapitre sur le travail pourrait être ici repris. L'exercice est aussi considéré comme une forme de toilette interne, comme une purification du corps et au début du siècle par voie de conséquence une purification de l'âme.

On notera qu'au cours du temps, en fonction des changements de programmes mais aussi des changements de pratiques corporelles dans la société, les techniques du corps proposées sont très différentes. On reconnaît, dans tous les cas, les vertus morales des exercices, en les rapportant à la propreté, d'une part, et, d'autre part, à l'éducation athénienne ou spartiate.

En tout état de cause, si les jeux français ou les exercices de type gymnastique construite sont très valorisés au début de la période, avec le mens sana in corpore sano et la statuaire grecque, et le sport critiqué pour ses excès à partir des années cinquante, ce sont les sports anglo-saxons qui trouvent davantage gré au regard des rédacteurs de manuels avec le mens fervida in corpore lacertoso (valorisé en son temps par le Baron Pierre FREDI DE COUBERTIN). Et si, au début du siècle, ce qui semble important pour les exercices du corps réside dans le fait de se soucier, par ce biais, de sa santé et de produire un travail physique - donc d'avoir un loisir sain (opposé à l'alcool et aux jeux d'argent) -, à partir du moment où le sport prend la place des méthodes construites ou ludiques, voire naturelles, la valeur centrale devient l'exploit et le dépassement de soi (le héros)².

À partir de la "sportification » de l'exercice, dans les manuels, ceux-ci rapprochent, de plus en plus, le courage de la pratique corporelle et le héros des temps modernes devient, de moins en moins, le Chevalier D'ASSAS et, de plus en plus, un coureur de $400 \mathrm{~m}$. aux Jeux Olympiques ou des alpinistes de l'extrême, dans l'Himalaya. Courage, dépassement de soi et sport deviennent, finalement, les facettes d'un même modèle moral. 


\section{Conclusion} contrôle des corps, de technologie de soi que l'on cherche à inculquer aux élèves. Ce système, sous la IIIe République, qui fit voter les lois sur la laïcité et instaura une école non confessionnelle aurait dû être laïque. Or, il n'en est rien, leur étude systématique montre que ce sont tout d'abord les préceptes chrétiens et plus précisément catholiques qui fondent les programmes de contrôle des corps et au début du XXe siècle, à travers eux, des âmes.

La disparition des devoirs envers Dieu des programmes scolaires conduira à quelques modifications des contenus, notamment sur la question du suicide, mais ne changera pas radicalement le fond. La plupart des préceptes correspondent à des péchés capitaux ou à des valeurs cardinales catholiques. Même l'exercice physique, que l'on pourrait penser laïque, est empreint de valeurs religieuses comme la purification de l'âme par le corps.

La disparition des manuels de morale au profit de manuels d'instruction civique et même de l'intégration de l'instruction civique dans le cadre des programmes d'histoire et géographie à partir des années 90 , dit peut-être la fin de cette vision du monde dans l'école et dans la société.

81 Au travers des manuels scolaires de morale se dessine un discours visant le contrôle de l'animalité au profit d'une humanisation, un discours qui va de la chair hurlante et pulsionnelle au verbe ascétique, qui va de la bête (animal et démon) à l'ange. Le processus de civilisation décrit par Norbert éLIAS (1973), initié au sein des communautés religieuses (THOMAS, 2003) ou dans le Civilitate morum puerilium d'ÉRASME, est valorisé dans les manuels de morale.

Par ailleurs, les contraintes corporelles proposées visant au contrôle de soi sont à la fois un système de contrôle social et de micro-pouvoir, voire de biopouvoir, ainsi qu'une lutte contre «La Souillure », corporelle et donc morale, visant à mettre en ordre la société ( DOUGLAS, 1971). La reprise quasi systématique des péchés capitaux religieux, en général, chrétiens et plus précisément catholiques (du moins ceux qui concernent le corps) et des valeurs cardinales, voire théologales montre, également, comment l'école de la République (laïque), se fonde sur un socle axiologique judéo-chrétien, et peut-être même strictement catholique.

Les graines plantées par Jean-Baptiste DE LA SALLE au XVIIIe et XIXe siècle ont, probablement, grandi dans la morale républicaine. 


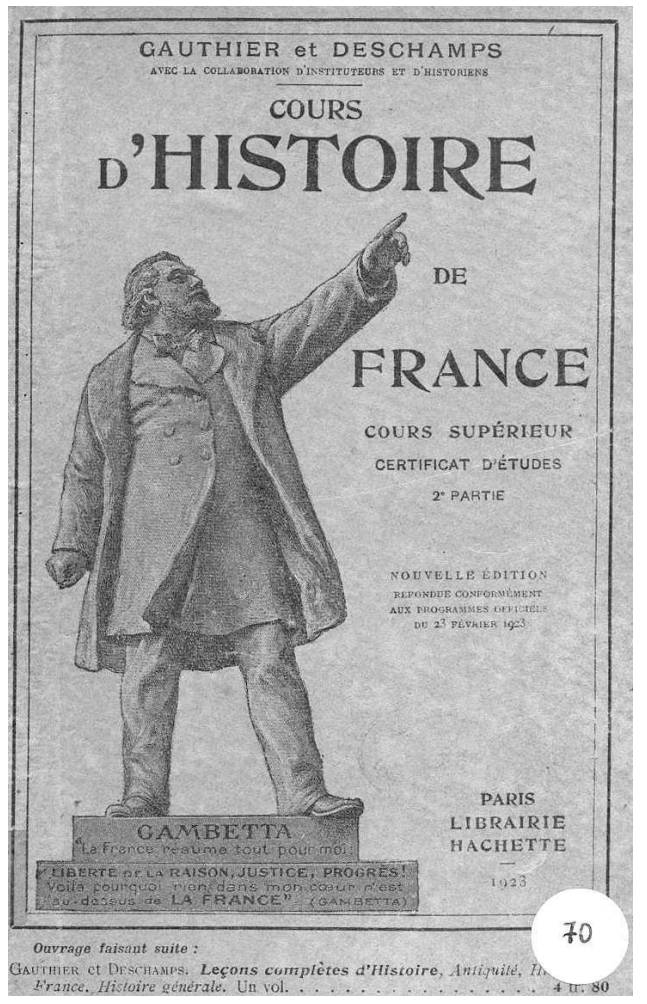

Cours d'histoire. Cours supérieur, certificat d'études, $2^{\mathrm{e}}$ partie, GAUTHIER et DESCHAMPS, Paris, HACHETTE, 1923. Source : CEDRHE.

\section{BIBLIOGRAPHIE}

Douglas (Mary), De la souillure. Essai sur les notions de pollution et de tabou. 1re Éd. 1965, Paris, MASPÉRO, 1971.

DURKHEIM (Émile), Le Suicide. Étude de sociologie, Paris, PAYOT, 1897.

ELIAS (Norbert), La Civilisation des mœurs, Paris, CALMANN-LÉVY, 1973.

ELIAS (Norbert), La Dynamique de l'Occident, Paris, CALMANN-LÉVY, 1975.

FOUCAULT (Michel), L'Archéologie du savoir, Paris, GALLIMARD, 1969.

FOUCAULT (Michel), Surveiller et punir, Paris, GALLIMARD, 1975.

GLEYSE (Jacques) et SOARES (Carmen), « Os manuais escolares franceses de educação física, de hygiene e de moral seriam sexistas ? (1880-2004) », Educação \& Sociedade, Campinas, Unicamp, janabril, 28, 102, 2008.

GLEYSE (Jacques), « Les représentations iconographiques des pratiques corporelles dans les Manuels d'Éducation physique, d'Hygiène et de Morale (1869 - 1935). La domination virile », in, L. DANIEL, dir., Actes du Colloques du CESH, Sport et Art dans l'Histoire, Lorient, CESH, 2008. 
FUMAT (Yveline), « La civilité peut-elle s'enseigner ? », Revue française de pédagogie, 132, juilletseptembre, 101-113, Paris, CNDP, 2000.

fumat (Yveline), Travail Propriété Pouvoir. L'idéologie des manuels de morale et instruction civique des débuts de la troisième république, 1881 à 1900. Thèse de sciences de l'éducation, Montpellier, non publiée, Montpellier III, 1984.

TAINE (Hyppolite), Derniers essais de critique et d'histoire. Paris, HACHETTE, 1894.

THOMAS (Jérôme), Corps violent, corps soumis. Le polissement des mœurs à la fin du Moyen-Age, Paris, L'HARMATTAN, 2003.

VERDELHAN-BOURGADE (Michèle), BAKHOUCHe (B.), BOUTAN (Pierre), ÉTIENNE (Richard), Les Manuels scolaires miroir de la nation?, Paris, L'HARMATTAN, 2007.

WEBER (Max), L'Éthique protestante et l'esprit du capitalisme, Paris, PLON, 1965.

\section{Archives utilisées pour l'article lui-même (sur 234 ouvrages étudiés) :}

BOURCEAU (E.) et FABRY (R.), Lectures expliquées avec enseignement moral et civique. Paris, Éditions de l'École, 1949.

BREMONT et MOUSTIER, (sans prénoms), L'Éducation morale et civique à l'école, Paris. DELALAIN, 1920.

CAZES (E.), Instruction morale et civique. Cours moyen, Paris, DELAGRAVE, 1890.

COMPAYRE (G.), Éléments d'instruction morale et civique, Paris, DELAGRAVE, 1883.

CURE (J.) et HOUZELLE (F.), Sommaires de leçons de morale à l'école primaire, Paris, E. BELIN, BELIN Fr, 1895.

DESPOIS (A.) et LABENNE (Mme), Lectures morales, Paris, DELAGRAVE, 1919.

DEVINAT (E.), Livre de lecture morale, Paris, LAROUSSE, 1920.

FOULON-LEFRANC (Mme), La Femme au foyer, Paris, Éditions de l'École, 1944.

HAUTIÈRE (de E.), Précis de morale pratique. Devoirs domestiques, devoirs civiques, etc., Paris, GARNIER Fr, 1887.

LAUNEy (H.), LAUNEy (J.) et RASCOL (L.), Morale. Instruction civique - Droit privé -Économie politique, Enseignement primaire supérieur, Paris, LAROUSSE, 1921.

MAILlet (A.), Pour devenir un homme, Paris, GEDALGE, 1928.

MEZIÈrES (M.-A.), Éducation morale et instruction civique à l'usage des Écoles primaires. Cours moyen et supérieur, Paris, DELAGRAVE, 1893.

NOËL (F.-A.), La Nouvelle lecture rationnelle. Premier livre de lecture courante. Leçons morales, leçons de choses, Paris, GEDALGE et Co, 1890.

NONUs (S.A.) et (P.C.), Résumés des leçons de morale et d'instruction civique. Amiens : POIRÉ-CHOQUET, 1890.

PAPE-CARPANTIER (M.) et DELON (Ch.), Petites lectures morales. Premières notions de grammaire, Paris, HACHETTE, 1900.

PECAUlt (É.) Cours de morale et d'hygiène, Paris, HACHETTE, 1882. 
PIERRE (A.), LETRAIT (L.), BODIN (Z.), Pour les petits. Lectures et récitations morales, Paris, NATHAN, 1909. PIERRE (A.), Minet (A.), MARTIN (A., Mlle), Nos Petits amis. Deuxième degré élémentaire, Paris, F. NATHAN , 1920.

POIGNET (A.) et BERNAT, (H.) Le livre unique de morale et d'instruction civique, Paris, Auguste GODCHAUX, 1919.

SEIGNette (A.), Morale et enseignement civique. Cours supérieur, Paris, P. DUPONT, 1895.

VILLARD (G.), Morale en action. Six à dix ans, Paris, NATHAN, 1964.

\section{NOTES}

1. Mes remerciements vont à Brigitte MORAND, précédente directrice du CEDRHE, à Hélène ANDRÉ et Evelyne ROBBEZ-MASSON qui ont grandement facilité ces recherches.

2. «Debout au seuil de mon 400 , je contemple cet étroit couloir brun marqué par ces deux minces rubans blancs entre lesquels ma vie va être enfermée pendant moins d'une minute [...] j'ai conscience d'avoir fait tout ce que j'avais à faire [...] Les lignes blanches si nettes, si pures, [...] m'emplissent le cœur d'une félicité immense [...] Elles vont contenir ce que des semaines, des mois durant, lentement, j'ai accumulé d'efforts vers la pureté morale et physique [je souligne...] je ne puis compter sur personne [...] contre mes concurrents je n'ai aucune haine aucune animosité, aucun sentiment bas qui viendrait déparer cette ambiance [...] cette confrontation va nous permettre de tirer du fond de notre corps, du fond de notre âme, le meilleur de nousmêmes » (VILLARD, 1961, p. 70-71).

\section{RÉSUMÉS}

La question de la laïcité et celle de la morale ont été récemment réactivées par les instances ministérielles en France, pour ce qui est de l'École. Le travail présenté, s'intéressant aux manuels scolaires de morale montre comment ces manuels publiés dans le cadre de l'École d'une République "laïque, indivisible, démocratique et sociale", sous l'égide du Ministère de l'Instruction Publique et des Beaux-Arts, du Ministère de l'Instruction ou du ministère de l'Éducation nationale, ne sont pas toujours aussi « laïcs » qu'on pourrait supposer qu'ils le soient. Le texte s'intéressera particulièrement à la question du corps dans ces manuels pour en constituer une sorte d'archéologie au sens de fOUCAULT.

The ministerial bodies in France, in terms of the school. The work presented, concerned with textbooks of moral shows how these books in spite of they are published as part of the School of a Republic "secular, indivisible, democratic and social", under the Department of Public Instruction and Fine Arts, Ministry of Education or the Ministry of Education, are not always as "secular" one might assume they are.

The text will pay particular attention to the question of the body in the manuals to be a kind of archeology in the way of FOUCAULT. 
INDEX

Mots-clés : corps, école, hygiène, laïcité, morale

Keywords : body, hygiene, moral, school, secularism

\section{AUTEUR}

\section{JACQUES GLEYSE}

Professeur des Universités en Sciences et Techniques des Activités Physiques et Sportives. Directeur du Centre de Documentation et de Recherche en Histoire de l'Éducation. LIRDEF, IUFM, Université Montpellier 2. 